\title{
The Role of Microtubule Associated Proteins in Plant Hypocotyl Elongation
}

\author{
Che Wang*, Xianling Wang, Qijiang Cao, Shuangtian Bi, Qiuyang Wang and Xinyu Liu \\ Department of Medicine, HE University School of Clinical Medicine, China
}

Received: January 17, 2018; Published: February 13, 2018

*Corresponding author: Che Wang, College of Biological Science and Technology, Shenyang Agricultural University, Shenyang, China, Tel: 86-24-88487163; Email: wangwangche@163.com

\begin{abstract}
The elongation of Arabidopsis hypocotyls is regulated by many factors such as light, phytohormones, transcription factors and the cytoskeleton. Microtubule is a member of cytoskeleton which plays an important role in hypocotyls elongation. The organization and dynamics of microtubules are regulated by microtubule associated proteins (MAPs). Recent years, several MAPs involve in the regulation of hypocotyls elongation mediated by upstream signals are identified. This review summarizes the recent progress and classifies the MAPs participate in the regulation of hypocotyls elongation into two categories, the positive and negative regulators.
\end{abstract}

Keywords: Microtubule associated proteins; Hypocotyls; Arabidopsis

Abbreviations: MT: Micro Tubule; MAPs: Microtubule Associated Proteins; COP: Constitutive Photomorphogenic; BR: Brassinosteroid; WVD: Wave Dampened; EIN: Ethylene Insensitive

\section{Introduction}

Hypocotyl is the part of a plant embryo or seedling plant that is between the cotyledons and the radicle or root. The regulation of hypocotyls elongation is crucial in the process of plant growth and development, because after the seeds germination, hypocotyls fully elongate to search of the soil surface and percept light then significantly inhibits hypocotyls elongation, the cotyledons unfold and the photosynthetic growth process could successfully begin. The Arabidopsis hypocotyls is widely used as a model for studies of cell elongation as hypocotyls cells elongate quickly without division during the postembryonic growth process [1,2]. Hypocotyl cells growth in the darkness start their elongation at the base and proceed in the acropetal direction reach their maximum length. The elongation of hypocotyls cells is regulated by both external and internal cues such as light, phytohormones, transcription factors, and the cytoskeleton [3-5].

Microtubule (MT) is one of the main members of cytoskeleton and plays important role in regulating cell expansion, division, and plant cell morphogenesis. Cortical microtubules regulate cell elongation by orientating cellulose fibrils and cellulose fibril arrays, thereby influencing the mechanical properties of the cell wall [6-9]. The orientations of cortical microtubules are associated with the growth status of etiolated hypocotyls $[10,11]$. The parallel array of cortical microtubules is dominantly transversely oriented to the hypocotyls longitudinal growth axis in elongating hypocotyls cells and longitudinally oriented when cell elongation stops [10], suggesting the regulation of the organization and dynamics of cortical microtubules is crucial for hypocotyls cell growth. The organization and dynamics of cortical microtubules is regulated by microtubule associated proteins (MAPs) [12]. In this article, plants MAPs involve in hypocotyls elongation are classified, on the basis of their function, into two categories: one is positive regulator and the other is negative regulator in the elongation of hypocotyls. In addition, we also discuss how these proteins mediate the synergistic and antagonistic effects in hypocotyls elongation.

\section{MAPs Play Positive Roles In Hypocotyl Elongation}

Until now, several MAPs play positive roles in regulation of Hypocotyl elongation have been identified. Arabidopsis SPIRAL1 (SPR1) encodes a plant-specific MT-localizing protein [13]. Plants over expressing SPR1 shows enhanced resistance to an MTdisrupting drug and increased Hypocotyl elongation. In addition, the site of high SPR1 expression moved up wards along darkgrown hypocotyls. The temporal and spatial distribution of SPR1expressing cells in hypocotyls generally matches the zone of rapid cell elongation [13,14]. Moreover, microtubule destabilizing protein $40(M D P 40)$ as a positive regulator involves in Brassinosteroid (BR) mediated Hypocotyl elongation by destabilizing cortical microtubules [15]. MDP40 is the target of Brassinazole-resistant1 (BZR1), a key transcription factor in BR signal transduction 
pathway, the expression of MDP40 is induced by BR treatment. $M D P 40$ primarily expresses in the upper region of the hypocotyls which is considered to be a fast growing region after $4 \mathrm{~d}$. MDP4O RNAi lines show the shorter etiolated Hypocotyl phenotype and the cortical MTs in the MDP4O RNAi cells are less sensitive to the oryzalin treatment. This study identifies a novel positive regulator in Hypocotyl elongation and reveals a mechanism involving BR regulation of microtubules through MDP40 to mediate Hypocotyl cell elongation [15].

\section{MAPs Play Negative Roles In Hypocotyl Elongation}

Besides positive regulators, there are also several MAPs that negatively regulate the elongation of hypocotyls. Recent years, a microtubule destabilizing protein 25 (MDP25) which is a calcium regulatory protein is identified as a negative regulator of Hypocotyl cell elongation [16]. MDP25 Overexpression results in shortening etiolated hypocotyls while mdp25 mutant lines have the opposite phenotypes. In addition, MDP25 is mostly expressed in the basal region of hypocotyls which is considered to be a non-growing region after $4 \mathrm{~d}$ of growth in the dark and the expression level of MDP25 in light grown hypocotyls is significantly higher than in dark. The localization of MDP25 is regulated by cellular calcium levels, increase calcium levels in cells cause MDP25 partially moves from the plasma membrane into cytosol, binds to and destabilizes cortical MTs.

This finding suggests that MDP25 link the change of cellular calcium levels mediated by light signal and cortical MTs in the regulation of hypocotyls elongation [16]. In addition, a recent study by Cao et al. identifies a novel Arabidopsis microtubule plus-end binding protein AUGMIN subunit8 (AUG8) that participates in inhibiting hypocotyls elongation by promoting the reorientation of cortical MTs [17]. The etiolated hypocotyls of aug8 are significantly shorter than WT. AUG8 binds to the plus ends of growing cortical MTs and promotes microtubule reorientation by regulating the dynamic instability of microtubule plus ends [17].

WDL3 and WDL5 belong to the microtubule-associated protein Wave-Dampened2 (WVD2)/WVD2-LIKE (WDL) family in Arabidopsis [18-21]. WDL3 involves in light inhibited Hypocotyl elongation through the degraded by the ubiquitin-26S proteasomedependent pathway in the dark [18]. Compare with wild type, WDL3 RNAi seedlings have longer while Overexpression seedlings have shorter hypocotyls in the light, however, the Hypocotyl of WT, WDL3 RNAi and Overexpression lines have no significant difference in the dark. The reason of different phenotypes of hypocotyls in the light and dark is that the WDL3 protein is abundant in the light but is degraded through the $26 \mathrm{~S}$ proteasome pathway in the dark [18].

In addition, Lian et al. analyzes the mechanism of WDL3 in the dark and finds that Constitutive Photomorphogenic 1(COP1) targets WDL3 for 26S proteasome-mediated degradation to control hypocotyls elongation in etiolated Arabidopsis seedlings. This study uncovers a function of COP1 in the cytoplasm to mediate hypocotyls elongation by regulating the abundance of MAPs WDL3 $[19,20]$. In darkness, ethylene inhibits etiolated Hypocotyl elongation as part of the well-known "triple response" [10]. A recent study shows that ethylene signaling participates in regulation of cortical microtubule reorientation and microtubule bundling plays important role in this process. The effects of ethylene on microtubule bundling are partially suppressed in a microtubule-bundling protein WDL5 knockout mutant (wdl5-1) [21]. Another study demonstrates that WDL5 plays a positive role in the phytohormones ethylene negatively regulated etiolated hypocotyls cell elongation. WDL5 as the directly target of Ethylene-Insensitive 3 (EIN3), a key transcription factor in the ethylene signaling pathway, inhibits the elongation of etiolated hypocotyls by stabilizing and reorientation cortical MTs [20].

\section{Conclusion}

In the pastyears, much progress has been made toward the MAPs involved in hypocotyls elongation. The elongation of hypocotyls is accurately regulated by positive and negative factors. Microtubule regulatory proteins function as positive or negative regulators that mediate hypocotyls cell elongation by altering microtubule organization and dynamics. In the process of hypocotyls elongation, the regulation of MAPs in not completely independent, on the one hand, MAPs are regulated by many internal or external signals involved in the signals mediated hypocotyls elongation, on the other hand, the stability of microtubules regulated by MAPs are important to the regulation of hypocotyls by the signal. In the future, as more MAPs involve in hypocotyls elongation mediated by different signals are identified, the mechanism of hypocotyls elongation regulation will be clearer.

\section{Acknowledgment}

We apologize to the authors whose work could not be included due to space limitations. This research was supported by the National Natural Science Foundation of China (No.31470358, 31500208, 31170232 and 31070157) and the startup funds from Shenyang Agricultural University (www.syau.edu.cn) to Xianling Wang.

\section{References}

1. Gendreau E, Traas J, Desnos T, Grandjean O, Caboche M, et al. (1997) Cellular basis of hypocotyl growth in Arabidopsis thaliana. Plant Physiology 114: 295-305.

2. Tsuchida Mayama T, Sakai T, Hanada A, Uehara Y, Asami T, et al. (2010) Role of the phytochrome and cryptochrome signaling pathways in hypocotyl phototropism. Plant Journal 62: 653-662.

3. Wang ZY, Nakano T, Gendron J, He J, Chen M, et al. (2002) Nuclear localized BZR1 mediates brassinosteroid induced growth and feedback suppression of brassinosteroid biosynthesis. Development Cell 2: 505513.

4. Ehrhardt DW (2008) Straighten up and fly right Microtubule dynamics and organization of non centrosomal arrays in higher plants. Current Opinion in Cell Biology 20: 107-116.

5. Niwa Y, Yamashino T, Mizuno T (2009) The circadian clock regulates the photoperiodic response of hypocotyl elongation through a coincidence mechanism in Arabidopsis thaliana. Plant Cell Physiology 50: 838-854.

6. Baskin TI (2005) Anisotropic expansion of the plant cell wall. Annual Review of Cell and Developmental Biology 21: 203-222.

7. Paredez AR, Somerville CR, Ehrhardt DW (2006) Visualization of cellulose synthase demonstrates functional association with microtubules. Science 312: 1491-1495. 
8. Somerville C (2006) Cellulose synthesis in higher plants. Annual Review of Cell and Developmental Biology 22: 53-78.

9. Lloyd C, Chan J (2008) The parallel lives of microtubules and cellulose microfibrils. Current Opinion in Plant Biology 11: 641-646.

10. Le J, Vandenbussche F, De Cnodder T, Van Der Straeten D, Verbelen JP (2005) Cell elongation and microtubule behavior in the Arabidopsis hypocotyls Responses to ethylene and auxin. Journal of Plant Growth Regulation 24: 166-178.

11. Crowell EF, Timpano H, Desprez T, Franssen Verheijen T, Emons AM, et al. (2011) Differential regulation of cellulose orientation at the inner and outer face of epidermal cells in the Arabidopsis hypocotyl. The Plant Cell 23: 2592-2605.

12. Buschmann H, Lloyd CW (2008) Arabidopsis mutants and the network of microtubule associated functions. Molecular Plant 1: 888-898.

13. Nakajima K, Furutani I, Tachimoto H, Matsubara H, Hashimoto T (2004) SPIRAL1 encodes a plant specific microtubule localized protein required for directional control of rapidly expanding Arabidopsis cells. The Plant Cell 16: 1178-1190.

14. Nakajima K, Kawamura T, Hashimoto T (2006) Role of the SPIRAL1 gene family in anisotropic growth of Arabidopsis thaliana. Plant Cell Physiology 47: 513-522.

15. Wang X, Zhang J, Yuan M, Ehrhardt DW, Wang Z, et al. (2012) Arabidopsis microtubule destabilizing protein 40 is involved in brassinosteroid regulation of hypocotyl elongation. The Plant Cell 24: 4012-4025.
16. Li J, Wang X, Qin T, Zhang Y, Liu X, et al. (2011) MDP25 a novel calcium regulatory protein, mediates hypocotyl cell elongation by destabilizing cortical microtubules in Arabidopsis. The Plant Cell 23: 4411-4427.

17. Cao L, Wang L, Zheng M, Cao H, Ding L, et al. (2013) Arabidopsis AUGMIN subunit8 is a microtubule plus end binding protein that promotes microtubule reorientation in hypocotyls. The Plant Cell 25: 2187-2201.

18. Liu X, Qin T, Ma Q Sun J, Liu Z, et al. (2013) Light regulated hypocotyl elongation involves proteasome dependent degradation of the microtubule regulatory protein WDL3 in Arabidopsis. The Plant Cell 25: 1740-1755.

19. Lin N, Liu X, Wang X, Zhou Y, Li H (2017) COP1 mediates dark-specific degradation of microtubule-associated protein WDL3 in regulating Arabidopsis hypocotyl elongation. Proceedings of the National Academy of Sciences 114(46): 12321-12326.

20.Sun J, Ma Q Mao T (2015) Ethylene regulates Arabidopsis microtubule associated protein WDL5 in etiolated hypocotyl elongation. Plant Physiology 169: 325-337.

21. Ma Q Sun J, Mao T (2016) Microtubule bundling plays a role in ethylene mediated cortical microtubule reorientation in etiolated hypocotyls. Journal of Cell Science 129(10): 2043-51.

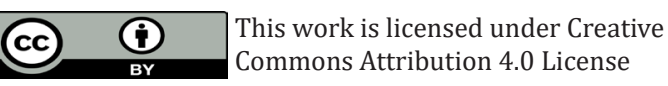

Submission Link: http://biomedres.us/submit-manuscript.php

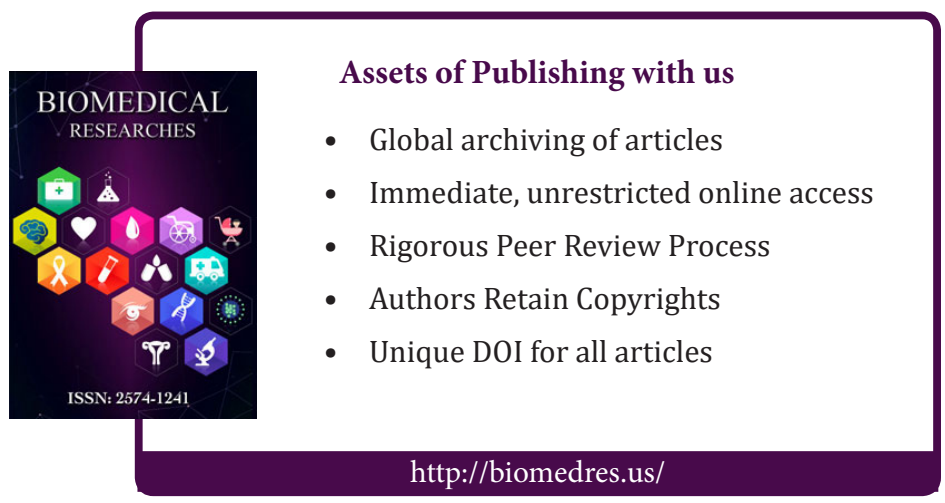

